\title{
A simple special-purpose interval-duration timer for acoustic research
}

\author{
JAMES M. LYNN \\ Ohio State University, Columbus, Ohio 43210
}

\begin{abstract}
A special-purpose interval-duration timer is described. The device is designed to interface with commercially available psychophysical packages, to be used in time interval calibration. The extremely low cost of materials justifies the construction of this essentially single-purpose device.
\end{abstract}

Gating of acoustic signals for auditory stimulus control typically is accomplished with commercially available electronic switches in conjunction with timebase generators (interval timers, one-shot multivibrators, and the like). ${ }^{1}$ Calibration of time intervals requires the use of expensive timer/counter devices. A specialpurpose interval-duration timer was designed for calibration of signal gating intervals. This timer frees the more expensive and sophisticated timer/counter devices for other tasks. The instrument described here interfaces with Coulbourn Instruments one-shot (Model S52-50) and universal (Model S53-21) interval timers, with the use of the computer output buffer (Model S62-06) or the FET buffer described later. The buffering arrangement is necessary in order to convert the $-12-\mathrm{V}$ logic of the Coulbourn Instruments apparatus to +5.V logic. The same buffering arrangement can be applied to Grason-Stadler 1200 systems, which also use $-12 \mathrm{~V}$.

Three factors were considered in design of this device: (1) timing accuracy, (2) simplicity of operation and construction, and (3) availability of materials. Design simplicity tends to limit the functional flexibility of the device, but it also reduces cost. Timing accuracy is not sacrificed, however. All materials for construction of the timer are readily available from local retail outlets.

\section{FUNCTION}

Figure 1 is a schematic design of the timing device showing the oscillator, gate, frequency divider, and counter. An accurate and stable time base is generated with the simple crystal oscillator shown in the upper left of Figure 1. It is constructed with one NAND gate from a 4011 CMOS integrated circuit. The oscillator produces a stable $1.0-\mathrm{MHz}$ signal that is gated by a second NAND from the 4011. A +5.V logical assertion (the buffered output from a Coulbourn Instruments or Grason-Stadler interval timer) on the countenable (CE) input to the gate allows the time-base signal to pass on to the next stage, a 2-decade biquinary frequency divider (7490a and $7490 \mathrm{~b}$ ). Each stage of the frequency divider produces a $50 \%$ duty-cycle square wave, the frequency of which is $10 \%$ of the input frequency. This 2-decade divider, there- fore, converts the $1.0 \mathrm{MHz}$ signal to a $10 \mathrm{kHz}$ time base. The fourth stage consists of apparatus for counting, decoding, and controlling the digital display of the time base. The unit counts pulses of the $10 \cdot \mathrm{kHz}$ signal for the duration of the assertion on the CE input of the gate and produces a five-digit display of the time duration, with seconds as the most significant digit. Outputs of five cascaded counters $(7490 \mathrm{c}-7490 \mathrm{~g}$ ) are decoded from BCD to seven-segment arrays (7448a7448 e) and displayed on five seven-segment LED digits. Only two of the counter stages are shown here; the remainder of the stages are wired exactly as the two shown in Figure 1.

\section{DESIGN CONSIDERATIONS}

With the exception of the 4011 , all components used in construction are from the TTL family of integrated circuits. These devices represent relatively old integratedcircuit technology; thus, they are simple, readily available, and quite inexpensive. The crystal oscillator and CE gate are constructed with the CMOS 4011 integrated circuit to facilitate design simplicity and oscillator reliability. Although the CMOS and TTL families usually are not mixed in circuit design, the 1,000-ohm "pulldown" resistor at the output of the CE gate provides adequate and simple interface. Note that the 4011 contains four NAND gates, only two of which are used in this device. The inputs to the remaining two gates (Pins 8, 9, 12, and 13) must be tied to ground. Also, the input to the $\mathrm{CE}$ gate (Pin 5) must be either asserted $(+5 \mathrm{~V})$ or negated (grounded). The readout will be erratic if this input is left unconnected.

The 7448 decoder/drivers are designed to drive common cathode LED displays. They are capable of driving most commercially available LED displays with no additional interface. The decoder-to-display connections are direct, rather than multiplexed. The direct-drive arrangement was selected for design simplicity.

It is necessary to manually reset all counters to zero before a new timing cycle is initiated. The reset (RE) function of the 7490 counters requires one SPST normally closed pushbutton switch, through which Pins 2 


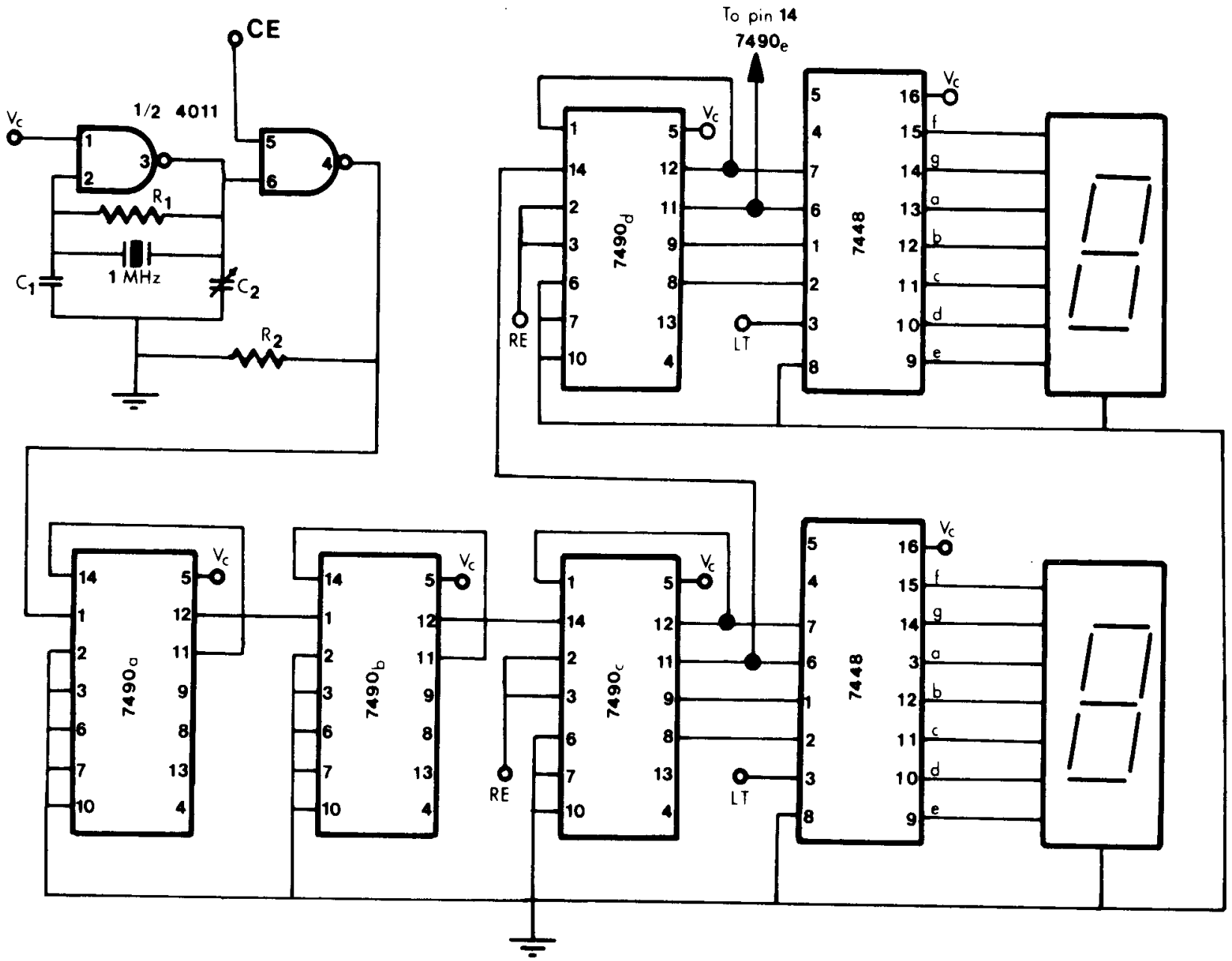

Figure 1. Schematic diagram of a digital interval-duration timer. Only the first two of five counting/display stages are shown. The remaining three stages are wired exactly as the first two. (R1: 10 megohms, R2: 1,000-ohm pull-down resistor; C1: 30 picoF, C2: variable from 7 through 50 picoF trims the crystal oscillator to $1.0 \mathrm{MHz}$. All resistors are $.25-\mathrm{W}$ resistors, and capacitors are $\pm 10 \%$ ).

and 3 of $7490 \mathrm{c}-7490 \mathrm{~g}$ are tied to ground. Use of the lamp-test (LT) function of the 7448 decoders is optional. It requires a normally open pushbutton switch, through which Pin 3 of each 7448 can be grounded momentarily. The LT function can be deleted from the device by connecting Pin 3 of each 7448 to the positive supply voltage.

The instrument requires a well regulated $+5 \cdot \mathrm{V}$ power supply capable of providing $.5 \mathrm{~A}$. A 7805 voltage regulator provides sufficient current and regulation.

\section{ACCURACY AND NOISE IMMUNITY}

Two sources of error are inherent in devices of this nature. Crystal oscillators are very stable, but they drift slightly with changes in temperature. A typical $1.0-\mathrm{MHz}$ crystal with temperature coefficient of $20 \mathrm{ppm} / 1^{\circ} \mathrm{C}$ is likely to drift $20 \mathrm{~Hz}$ with each $1^{\circ} \mathrm{C}$ of temperature change. This is equivalent to a timing error of $.02 \mathrm{msec} / 1^{\circ} \mathrm{C}$ in the present application.

Quantization errors occur when the CE gate is open for only a portion of the duty cycle for the least significant digit. In the present device, the error is $\pm .1 \mathrm{msec}$.
Although acceptable in many applications, this percent quantization error is considerably larger for the measurement of short time intervals. Percent error for measurement of a $1.0-\mathrm{sec}$ time interval is $\pm .01 \%$. Quantization error for measurement of a $10-\mathrm{msec}$ interval, however, is $\pm 1.0 \%$.

A potentially serious problem with TTL devices is noise, either on the input to a gate or on the power supply. Most manufacturers of TTL integrated circuits specify worst-case noise immunity of $.4 \mathrm{~V}$ (only $8 \%$ of supply voltage). Typical noise immunity values range from $.58 \mathrm{~V}$ (nearly $12 \%$ ) to $1.2 \mathrm{~V}(24 \%)$. Care must be taken, therefore, to minimize noise. If a 7805 voltage regulator is used in the power supply, it should be followed by a high-quality 1.0 -microF tantalum capacitor located as close to the 7805 as possible, and with the shortest possible leads. Additional noise immunity can be achieved by including one .01 - to .1-microF decoupling capacitor for every two integrated-circuit packages. These short-leaded disk capacitors should be distributed evenly throughout the system.

Unused inputs should be tied to ground. Also, circuit board layout should be designed to minimize the lengths 
of interconnection wires. In areas in which radiofrequency electromagnetic radiation is a problem, the unit may require a grounded metallic package as shield.

\section{DESIGN OPTIONS}

The unit can be modified to meet a number of special requirements. If the biquinary frequency dividers are wired as counters (note Pins 1 and 14) and the outputs are decoded and displayed, the device produces seven significant digits, with quantization error reduced to 1 microsec.

For individuals who do not have and do not wish to purchase a computer output buffer, a simple logic-level converter can be built with a single FET transistor, a $1,000-0 h m$ resistor, and an additional gate from the 4011 integrated circuit, as shown in Figure 2. A logical assertion from a Coulbourn Instruments device will produce $+5 \mathrm{~V}$ at the $\mathrm{CE}$ gate with this arrangement.

The basic counting circuit can be used for other functions with only slight modifications of the input configuration. The unit can be converted to time the interval between two events with the addition of a toggle flip-flop on the input. The first event sets the flip-flop and starts the timer. The second event resets the flip-flop and stops the counter. The toggle may be an external device such as the Coulbourn Instruments RS/T (Model S41-12) or a CMOS Type D (4013) or Type JK (4027) wired to toggle.

If Pin 6 of the NAND gate is fed by an external source and Pin 5 is held in an asserted state, the unit will function as a simple event counter. The external signal must have a rise time less than 5 microsec and have only one ground-to-positive transition per pulse. Rise times can be improved by buffering with a Schmitt trigger, such as the CMOS 4093 or 4584 . Again, inputs of unused gates must be tied to ground. With a calibrated $1-\sec$ assertion on Pin 5 of the NAND gate, the unit will count events per second.

Although we used TTL, the unit can be converted to CMOS and therefore made directly compatible with the $-12-\mathrm{V}$ logic of commercial instruments. Although this

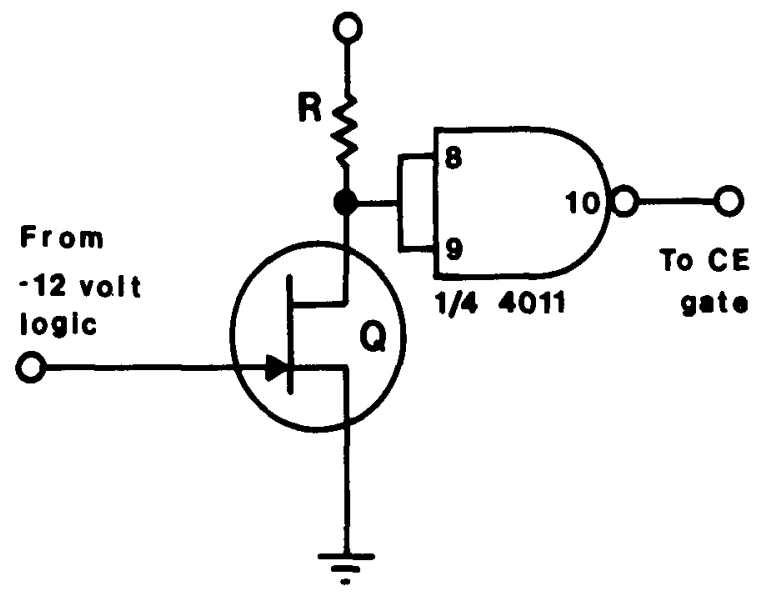

Figure 2. Alternative buffering arrangement to change the $-12-V$ logic of the Coulbourn Instruments devices to $+5-\mathrm{V}$ logic required for the interval-duration timer. (R: $1,000 \mathrm{ohms,}$ $\pm 10 \%, .25 \mathrm{~W}$; Q: RS-2035 or similar high-speed N-channel FET switching transistor.)

logic compatibility appears to be an advantage, difficulties in driving LED displays with CMOS integrated circuits increased circuit complexity and cost. If the device is built as shown and components are purchased from local retail outlets, the cost of electronic components is less than $\$ 30$. The cost of circuit board, sockets, and so on, depends on construction techniques.

\section{NOTE}

1. An alternative signal gate driver consists of a presettable down-counter and precision clock. By connecting the counter outputs to the gate control through an appropriate OR gate, the user can keep the signal gate open as long as the counter is counting down; it will close when the counter has counted out (down to zero). Signal duration, therefore, is set by the preset number times the period of the clock. A gate driver of this design tequires no calibration other than clock frequency. It does, however, require more expensive counter circuits and additional hardware for presetting the counters.

(Received for publication March 13, 1980; revision accepted May 15, 1980.) 